\title{
Les causes de la dégradation du chenal de Cotonou
}

\author{
Amoussa BADAHOUI ${ }^{1,2}$, Emile Didier FIOGBE ${ }^{1 *}$ et Michel BOKO ${ }^{2}$ \\ ${ }^{1}$ Unité de Recherche sur les Zones Humides, Département de Zoologie et Génétique, Faculté des Sciences et \\ Techniques, Université d'Abomey-Calavi, 01 BP 526 Cotonou, Bénin. \\ ${ }^{2}$ Ecole Doctorale Pluridisciplinaire "Espaces, Cultures et Développement', Faculté des Lettres, Arts et \\ Sciences Humaines, Université d'Abomey-Calavi, 01 BP 526 Cotonou, Bénin. \\ *Auteur correspondant, E-mail : edfiogbe@yahoo.fr, Tél. : 90948060
}

\section{RESUME}

Le chenal de Cotonou abrite sur son plan d'eau plusieurs infrastructures dont trois (3) ponts qui relient les deux rives, un barrage et des pêcheries sédentaires. On constate également que les rives du chenal sont occupées par de nombreux dépotoirs sauvages d'ordures ménagères. Les interventions humaines sur le chenal ont engendré de nombreux problèmes environnementaux dont les plus significatifs sont la pollution, l'érosion des berges et des fonds, et le comblement du plan d'eau. La dégradation du chenal de Cotonou provient de plusieurs facteurs principaux, notamment la pollution, sous toutes ses formes, qui a affecté la qualité des eaux, la construction du port en eau profonde avec l'ouverture permanente du chenal ayant entraîné d'importantes modifications hydrologiques, hydrobiologiques et socioéconomiques, la construction du barrage de Cotonou qui présente un défaut technique dans le mécanisme de réglage des échanges mer- lagune et, enfin, les activités de la pêche malgré son interdiction dans le chenal par les pouvoirs publics. D'autres facteurs secondaires sont identifiés parmi les causes de la dégradation du chenal tels que, le transport illicite des produits pétroliers et l'extraction de sable lagunaire. L'équilibre biologique de ce chenal, point focal de notre diagnostic, passe inévitablement par la mise en œuvre d'un plan d'actions qui vise à lutter contre la pollution des eaux, à assurer la régulation des échanges mer - lagune, à stabiliser les berges et à interdire la pêche dans le plan d'eau.

(C) 2009 International Formulae Group. All rights reserved.

Mots clés : chenal, Cotonou, pollution des eaux, équilibre écologique.

\section{INTRODUCTION}

Selon Larousse (2002), un chenal est un passage ouvert à la navigation entre un port, une rivière, un étang ou une mer et un océan ou une autre mer.

Le chenal de Cotonou communément et à tort appelé "lagune de Cotonou" est un plan d'eau qui relie le lac Nokoué, le plus grand et le plus productif du Bénin (15000 ha), à l'océan Atlantique, frontière Sud du pays. Une forte population plus ou moins déshéritée est installée aux abords immédiats de ses deux rives, dans des habitations pour la plupart précaires où manquent l'hygiène et toutes infrastructures d'assainissement de base (Roche International, 2000).
De nombreuses activités économiques sont menées autour de ce plan d'eau dont l'une des rives abrite, en particulier, le plus grand marché du Bénin, le marché international de Dantokpa (Figure 1). Les rives du chenal sont occupées par de nombreux dépotoirs sauvages d'ordures ménagères. Les exutoires de plusieurs collecteurs des eaux usées de la ville de Cotonou débouchent dans le chenal et le lac, rendant le degré de pollution très alarmant.

Parmi les activités économiques, figure également la pêche continentale qui revêt une importance particulière, notamment dans le Sud-Bénin (Fiogbé et al., 2009). Elle contribue pour 75 à $80 \%$ des débarquements totaux de poissons, $40 \%$ des consommations 


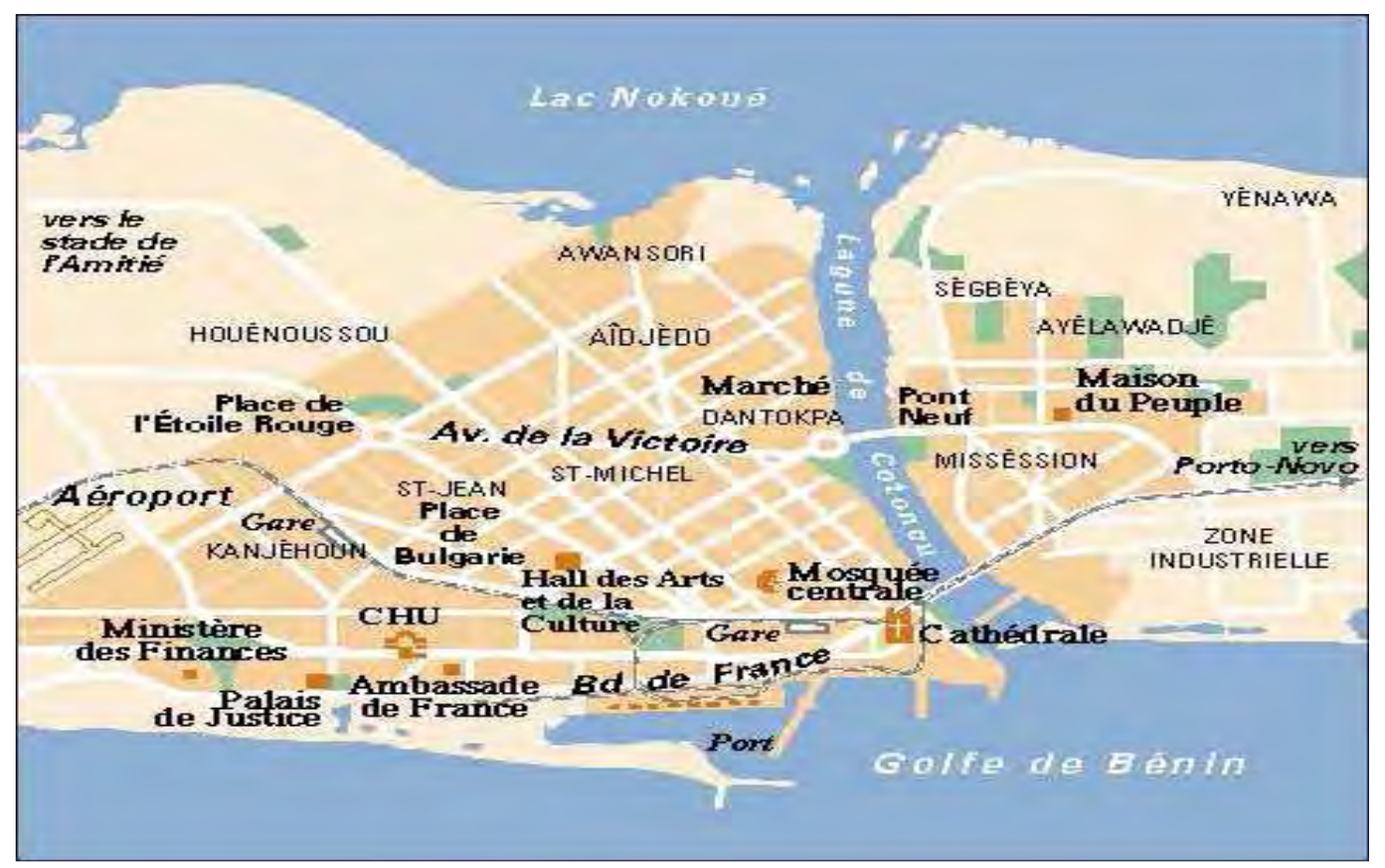

Figure 1 : Carte de la ville de Cotonou présentant la position de la lagune de Cotonou.

nationales de protéines animales et fait vivre plus de 600000 personnes (Roche International, 2000 ; Abou et al., 2007).

Certes, l'ouverture du lac Nokoué sur l'Océan Atlantique par le chenal de Cotonou lui confère une complexité avec une grande diversité des espèces halieutiques rencontrées dont l'une des caractéristiques est l'existence d'espèces à cycles biologiques anadrome, catadrome ou dia drome (Roche International, 2000).

Notons que l'influence de l'ouverture du lac Nokoué sur la mer s'étend également à la lagune de Porto-Novo qui communique avec le lac Nokoué par le canal de Totchè. C'est ainsi que le chenal de Cotonou a joué et continue de jouer un grand rôle dans le fonctionnement de cet écosystème dont l'équilibre a beaucoup varié dans le temps, suite aux différentes interventions de l'homme telles que la construction du port de Cotonou, la jetée de Placondji, les ponts de Cotonou et le barrage de Placondji.

En choisissant de réaliser cette étude, nous nous sommes fixés pour objectif de faire un diagnostic des facteurs de dégradation du chenal de Cotonou en vue de proposer quelques approches de solutions pour sa restauration écologique.

\section{MATERIEL ET METHODES}

La démarche méthodologique suivie pour la réalisation de cette étude repose sur une approche participative appuyée par les recherches documentaires et les résultats d'enquêtes de terrain effectuées par nousmêmes dans le chenal de Cotonou et le canal de Totchè servant de frontière naturelle entre le lac Nokoué et la lagune de Porto-Novo.

Pour une meilleure connaissance du chenal de Cotonou, nous avons exploité les résultats des analyses physico-chimiques et bactériologiques effectuées sous la direction de Bonou et Adisso (2002) sur le chenal de Cotonou et les caniveaux drainant les eaux de la ville. Les travaux du Bureau d'étude canadien Roche International ayant fait un diagnostic environnemental sur les plans d'eau du Sud-Bénin ont été aussi exploités. Plusieurs paramètres ont été étudiés dont principalement les propriétés physicochimiques de l'eau, la nature des sédiments, les communautés biologiques caractéristiques et la productivité de l'écosystème aquatique.

Les méthodes d'évaluation des pollutions physico-chimique et microbienne utilisées dans les études conduites sous la direction de Bonou et Adisso (2002) 
respectent les principes et précautions ciaprès:

- le choix raisonné de quatre sites de prélèvement a été fait pour mesurer le degré de pollution organique due en grande partie aux excrétas, aux eaux usées et aux ordures ménagères ;

- au total quatre campagnes d'échantillonnage ont été menées sur les stations retenues et les prélèvements ont eu lieu à des heures identiques de la journée, directement à partir de la barque avec un bécher de $500 \mathrm{ml}$ pour l'eau de surface et d'une bouteille de prélèvement pour l'eau de fond ;

la température, le $\mathrm{pH}$, la conductivité, l'oxygène dissous et la salinité sont mesurés in situ ;

- les échantillons d'eau sont ramenés au laboratoire pour l'analyse des sels dissous et des paramètres bactériologiques ;

- des précautions sont prises pour la conservation des échantillons afin d'éviter toute variation due aux conditions extérieures et toutes contaminations par des bactéries de l'air ;

- les flacons de prélèvement pour les analyses bactériologiques sont stérilisés à l'autoclave à 1,5 bars de pression et à la température de $120{ }^{\circ} \mathrm{C}$ pendant 15 minutes. Ces flacons sont au préalable bouchés au coton cardé et emballés dans du papier aluminium ;

- tous les échantillons sont conservés d'abord dans une glacière avec des conservateurs de froid puis au laboratoire à 4 ${ }^{\circ} \mathrm{C}$ dans un réfrigérateur jusqu'à l'analyse ;

- les analyses ont été effectuées dans les laboratoires de la Direction de l'Hydraulique et de la Direction de l'hygiène et de l'Assainissement de Base, respectivement pour la physico-chimie et la bactériologie.

Roche International (2000) a utilisé plusieurs méthodes pour mesurer les paramètres physico-chimiques de l'eau dont les plus importants sont :

- l'heure et la position géographique ont été mesurées à l'aide d'un appareil de positionnement GPS Tracker Magellan ;

- la profondeur a été mesurée au moyen d'une corde plombée calibrée;

- la transparence a été mesurée avec le disque de Secchi ;
- le prélèvement de l'eau a été effectué à une profondeur d'un mètre sous la surface au moyen d'une bouteille d'échantillonnage à fermeture par déclenchement. Lorsque la profondeur était moindre ou que le courant était très fort, l'eau a été prélevée tout juste sous la surface ;

- l'oxygène dissous a été mesurée avec un oxymètre de marque WTW, modèle 340-A et une sonde OxyCal 315 ;

- la température, la salinité et la conductivité ont été mesurées au moyen d'un appareil de marque YSI, modèle 33 ;

- le pH a été mesuré au moyen d'un pHmètre WTW ;

- la turbidité a été mesurée avec un turbidimètre de marque ORBECO 6 Hellige, modèle 966 ;

- les sédiments ont été échantillonnés sur une épaisseur d'environ $10 \mathrm{~cm}$ par un plongeur muni d'une bouteille en plastique. Le prélèvement a été effectué soigneusement de manière à ne pas perturber la structure verticale de l'échantillon. Dans presque tous les cas, les sédiments ont été prélevés exactement au même endroit que l'eau ; là où les courants étaient trop forts, ils ont été prélevés légèrement en aval, près de la rive et à environ un mètre sous la surface de l'eau ;

- dès leurs prélèvements, les échantillons ont été placés dans une glacière contenant de la glace pour une période maximale d'environ huit heures. Ainsi, tous les paramètres qui devaient être mesurés dans les 10 jours suivant le prélèvement de l'échantillon ont été analysés dans le laboratoire de la Direction des pêches du Bénin, tandis que tous les autres paramètres ont été mesurés au Canada, au laboratoire d'Envirolab, filiale de Roche.

\section{RESULTATS}

Les travaux réalisés sur le chenal de Cotonou signalent dans l'ensemble, les niveaux de pollution alarmants auxquels le chenal est assujetti du fait des actions anthropiques qui ont entraîné la dégradation de la qualité de l'eau du chenal.

Il ressort que les points échantillonnés par Roche International (2000) à Agbato, quartier situé au bord du chenal, présentent pour les paramètres bactériologiques, des taux supérieurs aux normes autorisées pour l'eau de boisson. Il a été enregistré des taux de 300.000 colonies/l de coliformes totaux ainsi 
que des taux de coliformes fécaux de l'ordre de 20.000 colonies/l. Il a été noté également des taux de streptocoques fécaux de l'ordre de 1000 colonies/l ainsi que 200 colonies/l d'entérocoques fécaux et la présence du pathogène Vibrio fluvialis.

Les études réalisées sous la direction de Bonou et Adisso (2002) sur le chenal de Cotonou et les caniveaux drainant l'eau de la ville ont montré une pollution bactériologique de ces eaux. Bien que se situant dans la gamme des seuils acceptables, ces teneurs révèlent une contamination d'origine fécale. Cette contamination a été mise en évidence non seulement par des ions sulfates, nitrates, nitrites et ammoniums que contiennent les échantillons d'eau et qui sont indicateurs de pollution fécale, mais aussi par la présence de germes que sont Escherichia coli, Clostridium sulfito- réducteur, Salmonella et entérobacters.

Les hydrocarbures totaux ont été dosés dans le chenal de Cotonou. Leurs concentrations sont passées de 0,1 ppm en 1986 à des concentrations comprises entre 8 et 100 ppm en 1999. La DBO (Demande Biologique en Oxygène) et la DCO (Demande Chimique en Oxygène) sont respectivement équivalentes à 6 et $93 \mathrm{mg} / \mathrm{l}$ et la matière organique totale est de $6000 \mathrm{mg} / \mathrm{l}$ (Soclo, 1999).

Les industries qui sont concentrées autour de Cotonou rejettent leurs effluents dans le chenal de Cotonou et contribuent ainsi à sa pollution chimique (Soclo, 1999).

Les deux principales sources de matières organiques et de nutriments proviennent des rejets domestiques et des industries de transformation des produits alimentaires. Or la production excessive de matières organiques entraîne une accumulation de boues, et le processus de minéralisation consomme tout l'oxygène dissous présent dans la colonne d'eau, ce qui provoque des hécatombes de poissons. C'est pourquoi on dit souvent que les polluants organiques sont «des déchets demandeurs d'oxygène » (Dejoux, 1988 ; Boko, 2004).

D'autres facteurs ont été identifiés comme sources de cette dégradation dont notamment, les conséquences de la construction du port de Cotonou en eau profonde et du barrage de Cotonou avec l'ouverture permanente du chenal, et, enfin, l'exercice des activités de la pêche.

\section{DISCUSSION}

Le chenal de Cotonou fait l'objet de plusieurs types d'exploitation par les populations riveraines qui contribuent à la dégradation de cet écosystème. Dans nos discussions, nous analyserons les causes des différents facteurs de dégradation identifiés dans le chenal de Cotonou et leurs impacts sur les ressources en eau.

\section{Dégradation due à la pollution des eaux du chenal}

Tout au long du chenal, on observe des zones de dépôt d'ordures, des zones aménagées, des infrastructures économiques, des points de rejet des eaux usées. Comment les actions anthropiques qui s'exercent sur le chenal contribuent-elles à sa dégradation?

En effet, la pollution par le dépôt des ordures ménagères et le rejet des eaux usées constitue une source de dégradation du chenal. L'ampleur de cette pollution illustrée par les Photos 1 et 2 dépasse les limites du tolérable.

\section{Niveau de pollution dans le chenal}

On entend par pollution des eaux la diminution de leur valeur économique et de leur fonction dans la biosphère par suite du déversement en leur sein de matières polluantes par l'action anthropique ou des phénomènes naturels (Colas, 1976; Dejoux, 1988). Mais selon les normes de l'OMS (Organisation Mondiale de la Santé), on considère qu'une eau est polluée lorsque sa composition et/ou son état sont altérés de telle sorte qu'elle se prête moins bien à l'une ou à l'autre ou à l'ensemble des utilisations pour lesquelles elle conviendrait dans son état naturel (Furon, 1963 ; Boko, 2004).

Le niveau de pollution dans le chenal peut toutefois refléter l'image que présentent les berges qui sont des zones privilégiées de dépôt d'ordures provenant de la ville de Cotonou. Si la ville de Cotonou à elle seule, produit annuellement environ 150.000 tonnes d'ordures ménagères contenant une grande proportion de matières putrescibles (Roche Internationale, 2000), et que, environ $4.750 .000 \mathrm{~m}^{3}$ d'eaux usées sont collectées par an dans la même ville, dont $72 \%$ proviennent des ménages et $28 \%$ des industries et services 

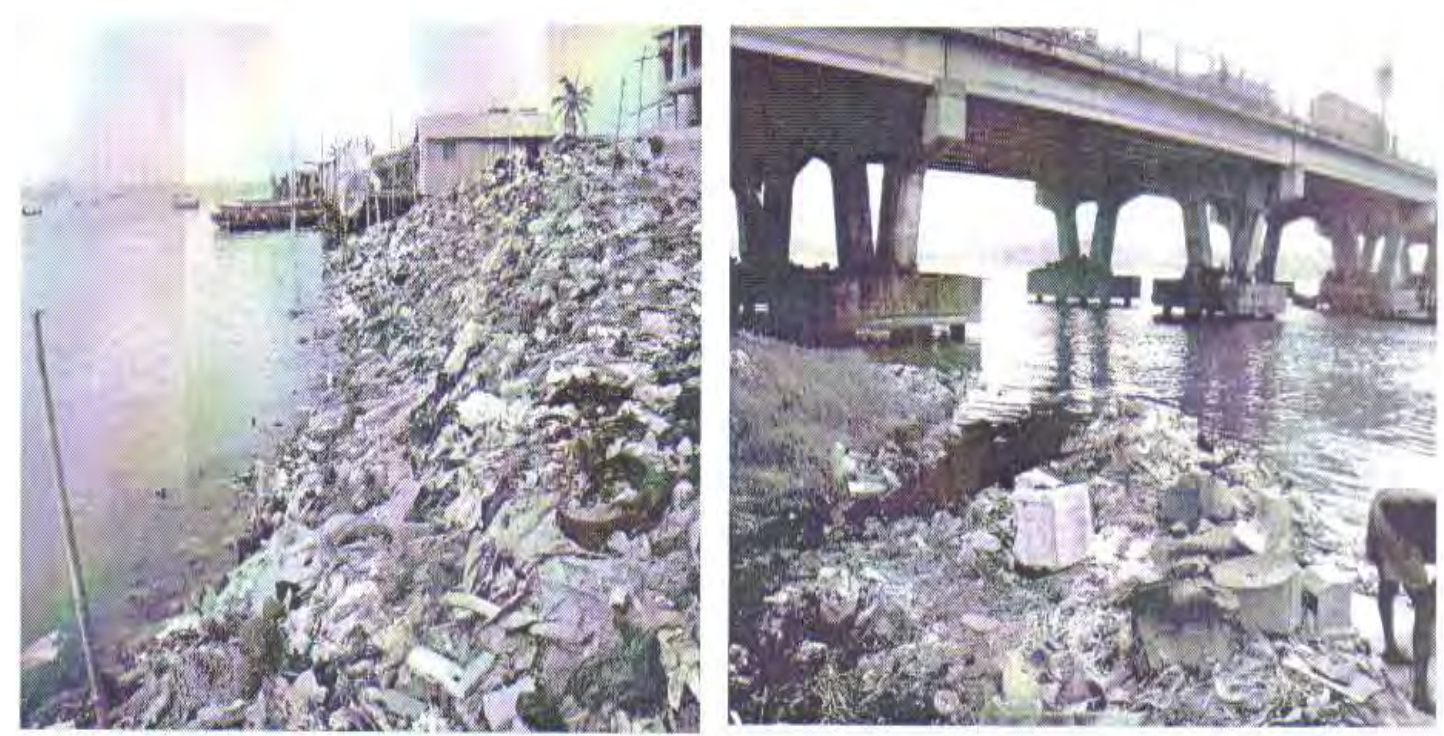

Photos 1 \& 2 : Dépotoirs sauvages le long du chenal de Cotonou - Dédokpo (Badahoui, 21 février 2008)

publics (Soclo, 1999); il est évident que les eaux du chenal soit polluées au contact de ces éléments nuisibles (Tableau 1). La construction sur le chenal de nombreuses latrines flottantes payantes (Photos $3 \& 4$ ), aggrave encore la pollution des eaux. Au quartier Agbato par exemple, on en dénombre plus d'une cinquantaine. Il est étonnant de constater que la pêche est pratiquée directement dans les environs immédiats de ces lieux, avec les nombreux risques de contamination par les métaux lourds des poissons qui sont pêchés. De même, les exutoires de plusieurs collecteurs d'assainissement de la ville de Cotonou qui sont aménagés dans le chenal rendent très élevé le degré de pollution des eaux drainées par les collecteurs (DBO5 très élevé de 243 mg/l). En 1996, le chenal de Cotonou a reçu $397.000 \mathrm{~m}^{3}$ d'eaux usées, soit 217 tonnes de DBO5 (Soclo, 1999).

La pollution des eaux du chenal provient également d'autres sources. Les déchets qui sont déposés comme remblais dans les zones marécageuses ou rejetés dans les caniveaux se retrouvent dans le chenal par les eaux de ruissellement. Une partie significative, voire importante des ordures ménagères collectées dans la ville de Cotonou est déversée tout au long du chenal. Ces ordures qui sont charriées dans le chenal au moment des inondations sont constituées essentiellement de matières putrescibles et elles contribuent au même titre que les eaux usées domestiques à la pollution organique et bactériologique des eaux du chenal. Les déchets proviennent également des usagers des marchés de Dantokpa, de Gbogbanou et de Missèbo. Ces marchés sont dans leur grande partie en contact avec le chenal de Cotonou. Des milliers de personnes qui y vivent et travaillent sont responsables de nombreuses activités allant de la gastronomie à la manufacture, en passant par le travail du cuir et la vente de divers articles. Ainsi, tôt le matin ou au crépuscule le soir, sont déversées dans le chenal des quantités énormes d'ordures ménagères, de restes alimentaires, d'eaux usées et d'excréta. Les populations ont construit elles-mêmes des latrines de fortunes faites de pailles ou de nattes, juste suffisantes pour protéger leur nudité. A l'intérieur de ces latrines, les individus défèquent sur du papier qu'ils jettent directement dans le chenal.

Quels sont les impacts de la pollution sur la qualité des eaux du chenal ?

Impacts de la pollution sur la qualité des eaux

Les paramètres physico-chimiques des eaux du chenal ont subi des modifications. 
Tableau 1: Quantités de déchets solides et liquides recueillies.

\begin{tabular}{llll}
\hline $\begin{array}{c}\text { Lieu de provenance } \\
\text { des déchets }\end{array}$ & Ordures ménagères & \multicolumn{1}{c}{ Eaux usées } & \multicolumn{1}{c}{$\begin{array}{c}\text { \% eaux usées } \\
\text { déversées dans le } \\
\text { chenal }\end{array}$} \\
\hline Ville de Cotonou & $\begin{array}{l}150000 \text { tonnes/an } \\
\text { dont une grande partie } \\
\text { est déposée sur les } \\
\text { berges du chenal }\end{array}$ & $\begin{array}{l}4750000 \mathrm{~m} 3 \mathrm{dont} \\
72 \% \text { provenant des } \\
\text { ménages et 28\% des } \\
\text { industries et services } \\
\text { publics }\end{array}$ & $\begin{array}{l}397000 \mathrm{~m} 3 \text { soit } 217 \\
\text { tonnes de DBO5 }\end{array}$ \\
\hline Réalisé à partir des données de Soclo (1999) et Roche International (2000)
\end{tabular}
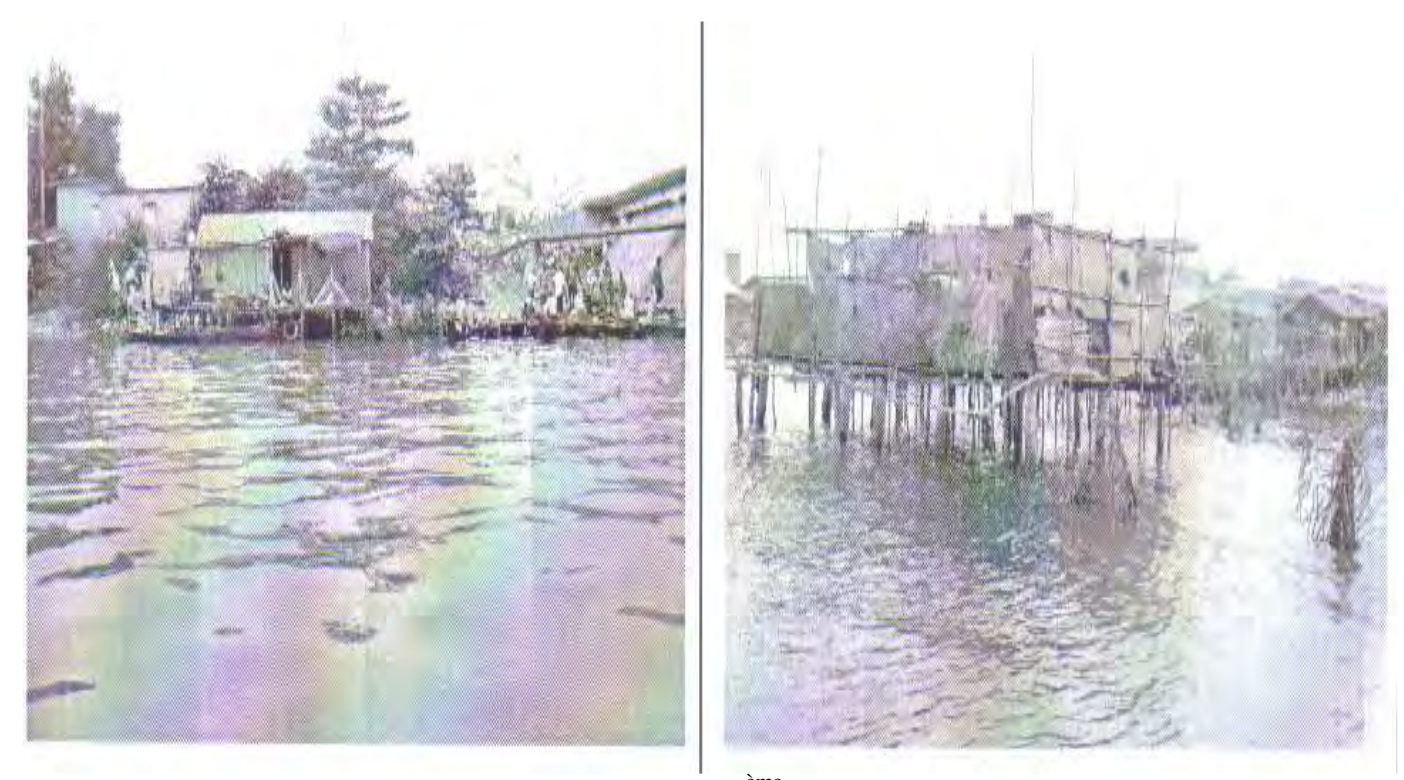

Photos 3 \& 4: Les latrines flottantes à côté du $2^{\text {ème }}$ pont côté marché Dantokpa (Photo 3) et au quartier Agbato (Photo 4) (Badahoui, 21 février 2008).

Les valeurs de température relevées dans le chenal sont comprises entre 28,5 et $30,8{ }^{\circ} \mathrm{C}$ (Bonou et Adisso 2002). Ses valeurs sont supérieures à la température de l'air ambiant qui est de $27{ }^{\circ} \mathrm{C}$. Si la température est un facteur qui conditionne véritablement l'abondance en êtres vivants animaux et/ou végétaux, la variation de quelques degrés de la température d'une eau peut être préjudiciable aux poissons par exemple ou à d'autres microorganismes importants dans la chaîne alimentaire.

Les valeurs du $\mathrm{pH}$ relevées dans le chenal de Cotonou se situent entre 7,3 et 8,55 (Bonou et Adisso, 2002). Ces valeurs qui sont supérieures à celles de l'eau de mer et des eaux brutes dont le $\mathrm{pH}$ de la plupart se situe entre 6,5 et 8,5 (Guilcher, 1959), seraient dues aux rejets des eaux usées par les collecteurs d'eau dans le chenal. Si par définition, le $\mathrm{pH}$ de l'eau mesure l'équilibre acide-base dans la plupart des eaux naturelles, il dépend surtout de l'équilibre dioxyde de carbone bicarbonate - carbonate. Le $\mathrm{pH}$ diminue lorsque la concentration en $\mathrm{CO}_{2}$ augmente et inversement. Or le pH relevé dans le chenal qui est supérieur à celui de l'eau de mer et des eaux brutes ne sera-t-il pas préjudiciable par exemple aux espèces dulçaquicoles ou anadromes?

Quant à la salinité de l'eau, les valeurs relevées dans le chenal vers l'embouchure (proche de la mer) sont plus élevées $(33,8$ et $32,85 \%$ ), tandis que celles relevées loin de l'embouchure sont de l'ordre de 30,05 et 31,33\%o (Soclo, 1999). La salinité qui indique la quantité de sels présents dans 
une masse donnée de solution, est un facteur très important qui conditionne la biodiversité. Elle varie avec la profondeur, la température, l'entrée ou la sortie des eaux marines (Marc, 1997). La salinité influence la conductivité électrique qui traduit la teneur globale des ions dans l'eau. La conductivité qui donne une idée sur la capacité d'une solution aqueuse à conduire le courant, varie suivant la concentration des électrolytes dans l'eau et surtout avec la température. (Le Barbe, 1993). Les taux de conductivité dans le chenal varient de 41,5 à $51,6 \mathrm{mS} / \mathrm{cm}$ (Bonou et Adisso, 2002). Les valeurs de conductivité notées dans le chenal de Cotonou qui sont supérieures à la valeur limite $(>500 \mu \mathrm{s} / \mathrm{cm})$ rapportée par Belaud (1987), signalent que les eaux du chenal sont très polluées dans leur ensemble.

Les gaz ont une importance cruciale pour le bien- être des espèces dans l'eau. Parmi les gaz, il y a l'oxygène pour les poissons, le $\mathrm{CO}_{2}$ pour les algues et le phytoplancton qui sont responsables de la photosynthèse. La majeure partie de l'oxygène dissous dans l'eau provient de l'air où il représente 20,95\% de l'air sec (Martin, 1985). Les valeurs relevées en oxygène dissous dans le chenal de Cotonou sont comprises entre 2 à $7,2 \mathrm{mg} / \mathrm{l}$ (Bonou et Adisso, 2002). Ces valeurs sont faibles à cause de la forte salinité des eaux, car plus une eau est saline, moins elle contient de l'oxygène dissous.

Au regard des nombreux impacts analysés plus haut, nous pouvons s'accorder avec Bonou et Adisso (2002) dont les études ont confirmé que les eaux du chenal de Cotonou sont sujettes à une pollution organique qui se manifeste sous diverses formes à savoir :

- milieu peu oxygéné et peu aéré ;

- cycle de l'azote perturbé (faible taux de nitrate et fort taux de nitrites) ;

- milieu oligothrophe et peu productif (faible taux de phosphates) ;

- forte charge en bactéries d'origine fécale correspondant à des valeurs élevées de DBO et de DCO.

La situation du chenal de Cotonou en matière de pollution est alarmante, et elle peut engendrer d'autres impacts négatifs sur les écosystèmes aquatiques. Cette pollution pourrait conduire à une baisse drastique de la productivité du chenal en général et des ressources halieutiques en particulier. Le risque de la disparition du plan d'eau par le comblement est à craindre car, le milieu devient de plus en plus anoxique. La pollution fécale pourrait constituer un autre danger pour la santé des populations humaines et en particulier pour les populations de pêcheurs, malgré que cette eau ne soit pas utilisée pour la boisson, mais qu'elle pourrait présenter des risques de contamination par la baignade surtout pour les pêcheurs et parfois certains riverains. Les nombreux déchets qui jonchent les berges du chenal ou qui sont déversés directement dans le chenal affectent les paramètres physico-chimiques du plan d'eau, et par conséquent la qualité de l'eau et les biocénoses. Ainsi, les pollutions organique et chimique découlant de ces déchets et les modifications hydro-sédimentologiques qu'elles engendrent, constituent les principaux facteurs de la dégradation de la qualité de l'habitat du poisson.

L'effet écologique de la pollution des eaux se fait sentir aux niveaux des organismes, de la population, de la biocénose et de l'écosystème. Au niveau des organismes, la pollution des eaux en particulier l'intoxication qu'elles occasionnent se définit avant tout sur la base de critères morphologiques et physiologiques (Gaujous, 1995). Ainsi, dans le cas d'une intoxication chronique par exemple, on observe souvent la naissance de formes anormales. La vitesse de croissance diminue chez les individus et la fécondité baisse. Sous l'effet de la pollution, le degré d'échange se modifie et la consommation ainsi que la conversion d'aliments diminuent. Les organismes changent de comportements et se déplacent vers les endroits où les conditions physicochimiques sont meilleures. Dans une intoxication aiguë, les organismes aquatiques changent leur manière de nager et se tiennent autrement dans l'eau. Par exemple, les mollusques s'enferment dans leurs coquilles et se recouvrent de mucus. Au niveau des espèces aquatiques, on observe lorsqu'il y a pollution de l'eau, des anomalies dans la structure et les interactions. Le nombre d'individus juvéniles diminue puisqu'ils sont plus sensibles à l'intoxication que les adultes. 
Le changement du sexe- ratio est caractéristique : on observe plus de mâles qui sont nains. La mortalité augmente et la natalité baisse.

\section{Dégradation du chenal due à l'aménagement des ouvrages portuaires}

Le chenal de Cotonou forme un couloir connecté au lac Nokoué par un entonnoir qui répartit le débit du chenal dans le lac au gré des flots et des jusants. Ce chenal est en fait un débouché artificiel creusé au XIX ${ }^{\mathrm{e}}$ siècle qui a subit beaucoup de modifications dans son fonctionnement hydrologique suite à la réalisation de certains ouvrages. On peut distinguer trois périodes dans le fonctionnement hydrologique du chenal.

\section{Période avant 1963}

Le premier ouvrage d'importance a été aménagé en 1885 par l'administration coloniale.

Selon Jean Pliya (1980), une tranchée de 1,50 $\mathrm{m}$ de large sur $1 \mathrm{~m}$ de profondeur a été faite par les Européens le 21 septembre 1885, suite à une inondation de Cotonou, ouvrant ainsi artificiellement le lac Nokoué sur la mer. Cette tranchée a atteint malheureusement 200 $\mathrm{m}$ en quelques heures et plus de $500 \mathrm{~m}$ quelques jours (Bourgoinie, 1972).

Selon Baglo (1980), l'étendue d'eau douce qu'était alors le chenal de Cotonou a donc été mise en communication avec la mer et, à partir de ce moment, on a assisté à des ouvertures et des fermetures périodiques de l'embouchure commandées par des effets de la dérive littorale et l'ampleur des crues de l'Ouémé. Avant cette ouverture, le niveau du lac Nokoué en période de crue dépassait de $2,4 \mathrm{~m}$ celui de la mer et sa superficie atteignait 45000 ha. L'évacuation de la crue vers la mer se faisait entièrement via la lagune de PortoNovo puis le chenal de Lagos. Après l'ouverture, l'eau de la crue de l'Ouémé se déversait directement du lac Nokoué à la mer par le chenal de Cotonou, drainant même les eaux de la lagune de Porto- Novo via le canal de Totchè. Puis, lors des périodes d'étiage de l'Ouémé, l'intrusion de l'eau salée à la faveur des courants de marée a aussi créé une modification majeure du régime hydrologique du chenal de Cotonou.

En 1959, l'ouverture artificielle du chenal de Cotonou suivie en 1960 de la construction du port de Cotonou a aussi engendré des perturbations importantes de la circulation littorale, lesquelles ont modifié profondément le régime hydrologique du chenal et du lac Nokoué.

Avant la construction du port de Cotonou, il arrivait que la dérive littorale referme l'ouverture du chenal après avoir permis la pénétration d'importantes masses d'eaux marines. En saison sèche, on observait alors une sursalinisation à cause de l'évaporation (les eaux atteignant parfois $38 \%$ o de salinité) et une concentration des sels nutritifs provenant des alluvions de l'Ouémé, ce qui aurait favorisé la productivité primaire (Baglo, 1994). Parfois, la crue n'arrivait pas toujours à ouvrir le chenal et elle était alors évacuée via la lagune de Porto- Novo et le chenal de Lagos. Lorsque la crue parvenait à être évacuée par le chenal de Cotonou, la dérive littorale refermait rapidement l'ouverture de sable, limitant ainsi grandement l'intrusion d'eau salée dans la lac Nokoué (Roche International, 2000).

On peut retenir globalement qu'avant la construction du port de Cotonou et de son épi Est, le chenal de Cotonou et le lac Nokoué se comportaient comme une lagune fermée à l'étiage et ouverte à la crue. A l'étiage, le niveau de la mer était moyennement plus élevé que le niveau du lac. La salinité est généralement moyenne dans le lac du fait de l'apport quoique faible des eaux douces des cours d'eau et légèrement plus élevée dans le chenal du fait de la proximité avec la mer. On observe dans le lac un gradient de salinité orienté d'Ouest en Est. A la crue, le niveau du lac et du chenal est supérieur à celui de la mer jusqu'à la rupture du cordon lagunaire de l'embouchure. Le niveau d'eau dans le lac et le chenal dépendra de l'importance de la crue et de la largeur de l'embouchure. Les eaux sont dès lors essentiellement douces.

\section{Période 1963- 1977}

Pendant la période coloniale, le Bénin disposait d'un Wharf qui sert de lieu d'amarrage pour les navires de commerce.

En 1948, devant l'incapacité du Wharf de faire face au commerce extérieur béninois, les autorités ont senti la nécessité de construire un port. Le littoral béninois étant affecté par la houle et le courant de Cotonou, on devrait donc tenir compte de ces facteurs dans la conception et la réalisation de 
l'ouvrage. Il a été donc adopté un modèle de port à accumulation de sable.

Dès 1963, avec la construction du port, le chenal de Cotonou est quasi ouvert en permanence (Baglo, 1994).

\section{Construction en eau profonde du port de Cotonou}

A partir du 13 novembre 1959 au $1^{\text {er }}$ août 1965, il y a eu des travaux de construction du port occasionnant à cet effet depuis 1961 une ouverture permanente du chenal de Cotonou, du fait de la nouvelle orientation des courants et des houles (Pliya, 1980). Le port de Cotonou construit en eau profonde est constitué d'un certain nombre d'ouvrages comprenant une jetée Ouest en enrochement longue de 1424 mètres, et une traversée Est en palplanches longue de 770 mètres qui ferme la zone portuaire à l'Est tout en laissant une passe d'entrée large de 180 mètres (Leite, 2002). Le rôle de la jetée Ouest en enrochement est de protéger la zone portuaire contre les effets de la houle et du courant de Cotonou, entre autres l'ensablement et les vagues. Elle devrait permettre également aux bateaux d'accoster sans risque de chavirer.

Les travaux de construction ont débuté en décembre 1960. Dès la construction des premiers mètres de cette jetée, d'importantes perturbations ont commencé par se faire sentir sur tout le littoral dont la plus remarquable est celle de l'arrêt de la dérive littorale. La jetée Ouest dont le rôle est d'empêcher l'ensablement va plutôt bloquer chaque année environ $1500000 \mathrm{~m}^{3}$ de sable (Leite, 2002). On assiste alors à un arrêt quasi-total de l'apport de sable à l'Est et à une accumulation de sable à l'Ouest de la jetée. Les conséquences immédiates de cet arrêt sont l'ouverture permanente du chenal de Cotonou qui a entraîné d'importantes modifications hydro biologiques et socio-économiques (Baglo, 1980).

Impacts de la construction du port sur le régime hydrologique du chenal

Depuis la construction du port, l'ouvrage qui est essentiellement responsable des perturbations hydrologiques est la jetée Ouest, perpendiculaire à la direction de la houle (Baglo, 1980). En bloquant la dérive littorale de sable, la jetée Ouest entraîne la déposition du sable du côté Ouest et, privant le courant côtier de son sable, elle en augmente la capacité de charriage. Les courants ramassent donc le sable du côté Est, entretenant ainsi l'ouverture du chenal de Cotonou (Roche International, 2000). Cette ouverture permanente du chenal permet donc l'intrusion périodique d'eau salée dans le lac Nokoué et, via le canal de Totchè, dans la lagune de Porto-Novo. Cela a donc augmenté considérablement les échanges avec la mer et l'exportation de sédiments, comme on peut le constater avec le déchaussement et l'affouillement des piliers de l'ancien pont d'Akpakpa (Roche International, 2000). De plus, l'ouverture permanente du chenal de Cotonou aurait alors entraîné une diminution importante de la productivité primaire par suite, d'une part, de l'évacuation des nutriments et des particules fines qui, auparavant, demeuraient dans le lac et, d'autre part, de l'action de brassage des vents et des courants de crue, de marée et de densité qui aurait augmenté la turbidité (Baglo, 1980). Cette ouverture permanente du chenal a favorisé également la pénétration massive des eaux marines accompagnées d'une élévation du taux de salinité qui variait de 0 à $30 \mathrm{~g} / \mathrm{l}$, occasionnant par là un appauvrissement du milieu en planctons et une invasion d'espèces animales d'origine marine et l'élimination de la flore et de la faune dulçaquicole (Troadec. 1990). A tout cela, s'ajoute la détérioration des activités de pêche avec une baisse de la production du lac Nokoué et de la lagune de Porto-Novo de 4000 tonnes environ entre 1960 et 1964 (Leite, 2002). Cette situation a entraîné également une baisse considérable du rendement de la pêche dont les captures sont passées de 15000 tonnes en 1959 à 5600 tonnes en 1969, puis à 4910 tonnes en 1975 (Baglo, 1980). On peut donc dire que la modification du régime du lac Nokoué et de la lagune de Porto-Novo a été en grande partie la cause de la baisse des captures dans ces plans d'eau au cours de cette période (Roche International, 2000). L'ouverture permanente du chenal de Cotonou a été particulièrement préjudiciable à l'équilibre écologique des complexes lagunaires du Sud-Bénin. Elle a entraîné la salinisation des eaux qui a pour conséquence la modification de la composition spécifique du stock halieutique et la disparition d'espèces strictement dulçaquicoles telles que Lates niloticus et 
Heterotis niloticus (Baglo, 1980). De même, l'ouverture permanente du chenal a entraîné d'une part, une diminution importante de la productivité primaire, et l'évacuation des nutriments et des particules fines d'autre part. On constate une augmentation de la turbidité des matières en suspension sous l'effet conjugué des vents et des courants de crue, de marée et de densité (Baglo, 1980).

En définitive, l'ouverture permanente du chenal a modifié radicalement le régime de salinité du lac Nokoué et, par voie de conséquence, sa faune aquatique. Mais selon Baglo (1980), c'est le régime de salinité qui a été radicalement modifié, plutôt que le niveau de salinité ; celle-ci se faisant sentir pendant une période bien plus longue, la période d'eau douce étant maintenant limitée à la période de crue et de décrue. Ce nouveau régime a effectivement entraîné une augmentation relative des captures d'espèces marines telles que Ethmalose, Chrysichtys, les huîtres et les crevettes, ainsi qu'une diminution relative des tilapia et des mugilidés (Hodonou, 1971 ; Roche International, 2000). De plus, ce prolongement important de la période de salinité et la restriction de la période d'eau douce a favorisé la prolifération et la survie des tarets, lesquels dégradent les branchages des acadjas, les pilotis des maisons et les pirogues (Baglo, 1980). De même, la productivité halieutique a chuté considérablement, la superficie des acadjas étant passé de 511 ha en 1959 à 186 ha en 1969 (Baglo, 1980).

Face aux dégâts occasionnés par la construction du port, les autorités Béninoises ont décidé d'édifier un barrage sur le chenal de Cotonou afin de rétablir l'équilibre écologique du complexe.

\section{Période 1977 à nos jours}

Elle correspond à la période où une digue en enrochement a été érigée à l'embouchure du chenal de Cotonou. Cette digue visait à réguler les échanges entre le chenal et la mer par une réduction de la section d'échange. Mais apparemment l'ouvrage n'a pas donné satisfaction aux populations de pêcheurs qui ont procédé à son écrêtement (Roche International, 2000).

Construction du barrage (digue) de Cotonou

En 1977, afin de limiter les conséquences reliées à l'ouverture permanente du chenal de
Cotonou, un barrage en enrochement a été construit dont les objectifs selon Baglo (1980), étaient de :

- éviter l'inondation de la ville de Cotonou ;

- protéger les trois ponts en réduisant la vitesse des courants ;

- ralentir la vitesse de la décrue afin de prolonger la période de reproduction et de croissance des espèces d'eau douce ;

- permettre une certaine intrusion d'eau salée afin de réduire les effets de la pollution des eaux.

Selon Leite (2002), ce barrage était construit d'une digue d'environ $420 \mathrm{~m}$ et de 6 pertuis de 4,5 $\mathrm{m}$ de largeur. L'ouvrage comporte un mécanisme de réglage des échanges mer-lagune. Mais il est précisé dans l'étude de faisabilité que la côte d'arasement de la digue en enrochements devrait être de 1,10 mètres. Or, le barrage réalisé est plus haut que celui proposé de 0,32 mètres. Les échanges furent totalement coupés en cours de travaux par une flèche littorale qui s'est reconstituée en avant du barrage. Cette fermeture indésirable a rendu inefficace le maniement du système de réglage. Avant même la fin des travaux, la construction de la digue avait déjà modifié la trajectoire des courants à l'embouchure, ce qui a entraîné le prolongement vers l'ouest de la flèche de sable existante jusqu'à refermer complètement l'embouchure en mai 1978.

Baglo (1980), a signalé l'apparition naturelle d'une flèche littorale de sable derrière le même barrage du fait de la réduction significative du courant lagunaire, avant même la fin de sa construction. Cette flèche de sable a pris progressivement de hauteur, entraînant de ce fait la fermeture progressive et totale du chenal le 6 mai 1978, avec les conséquences biologiques plus catastrophiques que les étapes précédentes (Photo 7).

Impacts environnementaux engendrés par la construction du barrage de Cotonou

Les impacts remontent dans les années 1978 et 1979, avec la fermeture du barrage qui a causé tour à tour des inondations importantes au cours des périodes de crue, entrecoupées d'étiages sévères (Baglo, 1980). De plus, cette fermeture a créé un milieu favorable à la prolifération du benthos et du phytoplancton, donnant la couleur verte des 
eaux du lac Nokoué et vert- crayeuse de la lagune de Porto-Novo (Baglo, 1980). Enfin, la crête du barrage a été construite à la côte d'arasement de 0,90 m IGN. Or, cette côte était supérieure de $0,5 \mathrm{~cm}$ à celle utilisée dans l'étude de faisabilité, ce qui pourrait avoir diminué significativement sa capacité de contrôle des eaux (Baglo, 1980). En août 1979, on a procédé à l'ouverture artificielle de la flèche de sable et laissé les pertuis ouverts en permanence, ce qui a entraîné à nouveau des perturbations importantes du régime hydrologique du lac Nokoué. Après une baisse sensible du niveau du lac, les pluies de 1979 ont longtemps retardé le retour à un niveau normal avant que l'étroite ouverture dans le barrage ne permette la décrue. On constate que la salinité en période d'étiage n'a jamais atteint les maxima d'avant le barrage, le seuil d'enrochement constituant une entrave à la pénétration de l'eau salée et favorisant l'évacuation d'une partie des crues par le canal de Totchè (Baglo, 1993). Cela a pour conséquence de réduire la vitesse des courants dans le chenal de Cotonou, diminuant ainsi l'érosion du fond (Baglo, 1980). Par contre, la côte de la crête du barrage étant supérieure à ce qui avait été prévu, on a observé une augmentation sensible de la vitesse des courants et de l'érosion des berges dans le canal de Totchè en direction de la lagune de Porto- Novo, causant ainsi un comblement à l'ouest de la digue du pont de Porto- Novo et limitant l'intrusion d'eau salée en provenance du chenal de Lagos (Baglo, 1980).

En résumé on peut retenir qu'à la suite de la construction du barrage de Cotonou en 1977, laquelle a entraîné la fermeture de l'embouchure, la salinité du lac Nokoué a été réduite considérablement, redevenant sensiblement la même qu'avant la construction du port. Une fois le barrage rouvert, en 1979, le régime de salinité d'avant la fermeture s'est approximativement rétabli. Toutefois, en raison de l'étroitesse du passage offert par les pertuis, la salinité maximale moyenne dans le chenal de Cotonou est passée de $23 \%$ à $33 \%$, alors qu'elle a diminué fortement dans les zones du lac plus éloignées du barrage, ce qui a eu pour conséquence de détruire une partie des mollusques marins qui s'y étaient établis. Par contre, le milieu étant moins propice aux tarets et plus favorable aux espèces d'eau douce et saumâtre, la superficie des acadjas et les captures ont commencé à augmenter.

Par ailleurs, il faut remarquer que les activités de pêche ne favorisent pas la restauration écologique du chenal de Cotonou.

\section{Dégradation du chenal due aux activités de pêche}

Les activités de pêche sont courantes dans le chenal de Cotonou. Outre les pêcheurs riverains, il y a d'autres pêcheurs professionnels provenant des villages lacustres du lac Nokoué qui viennent pêcher. Parmi les engins et techniques de pêche utilisés, certains sont sources de conflits parfois sanglants entre pêcheurs eux-mêmes. C'est le cas du filet Mèdokpokonou pratiqué par environ 3295 pêcheurs recensés dans vingt et un (21) villages sur le lac Nokoué, la lagune de Porto-Novo et le chenal de Cotonou (Alapini, 2001 ; Bonou et Adisso, 2002). En raison du caractère dévastateur de cet engin, son utilisation est interdite sur l'ensemble des plans d'eau de la République du Bénin, par Décret $\mathrm{N}^{\circ} 98-522$ du 5 novembre 1998.

Aussi, convient-il de signaler que par arrêté $\mathrm{N}^{\circ} 068 / \mathrm{MDR} / \mathrm{DC} / \mathrm{CC} / \mathrm{CP} / \mathrm{du} 12$ mars 1997, portant réglementation de la pêche sur le complexe Delta de l'Ouémé-lagune de Porto-Novo-lac Nokoué, et en son article 20, les pouvoirs publics ont interdit la pêche sur le chenal de Cotonou, afin de permettre à ce plan d'eau de jouer son rôle d'échange entre la mer et le lac Nokoué.

Malgré la prise de ces mesures réglementaires pour assurer une gestion durable des ressources halieutiques, la pêche est et demeure une activité qui s'exerce de façon permanente dans le chenal de Cotonou par des pêcheurs professionnels (Photos $5 \&$ 6). Au cours de notre enquête-cadre réalisée le jeudi 21 février 2008, nous avons recensé plusieurs campements de pêche installés sur les berges du chenal, des embarcations et des pêcheries sédentaires. Dans ces campements à l'image des taudis, les occupants en l'occurrence les pêcheurs n'ont pas accès à l'eau potable, à un logement durable ni à une surface habitable suffisante. L'assainissement et la sécurisation foncière des lieux sont 


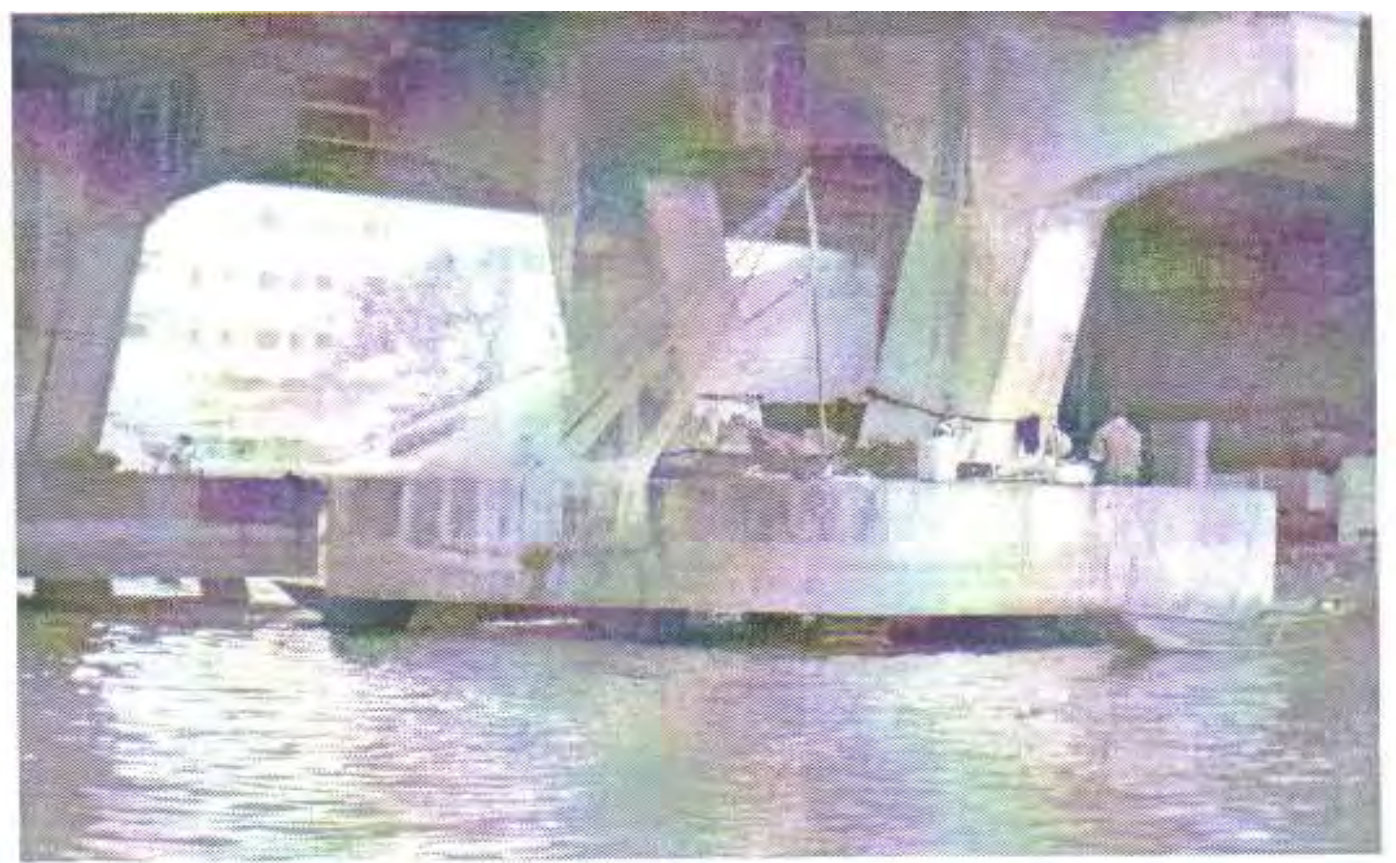

(Photo 5)

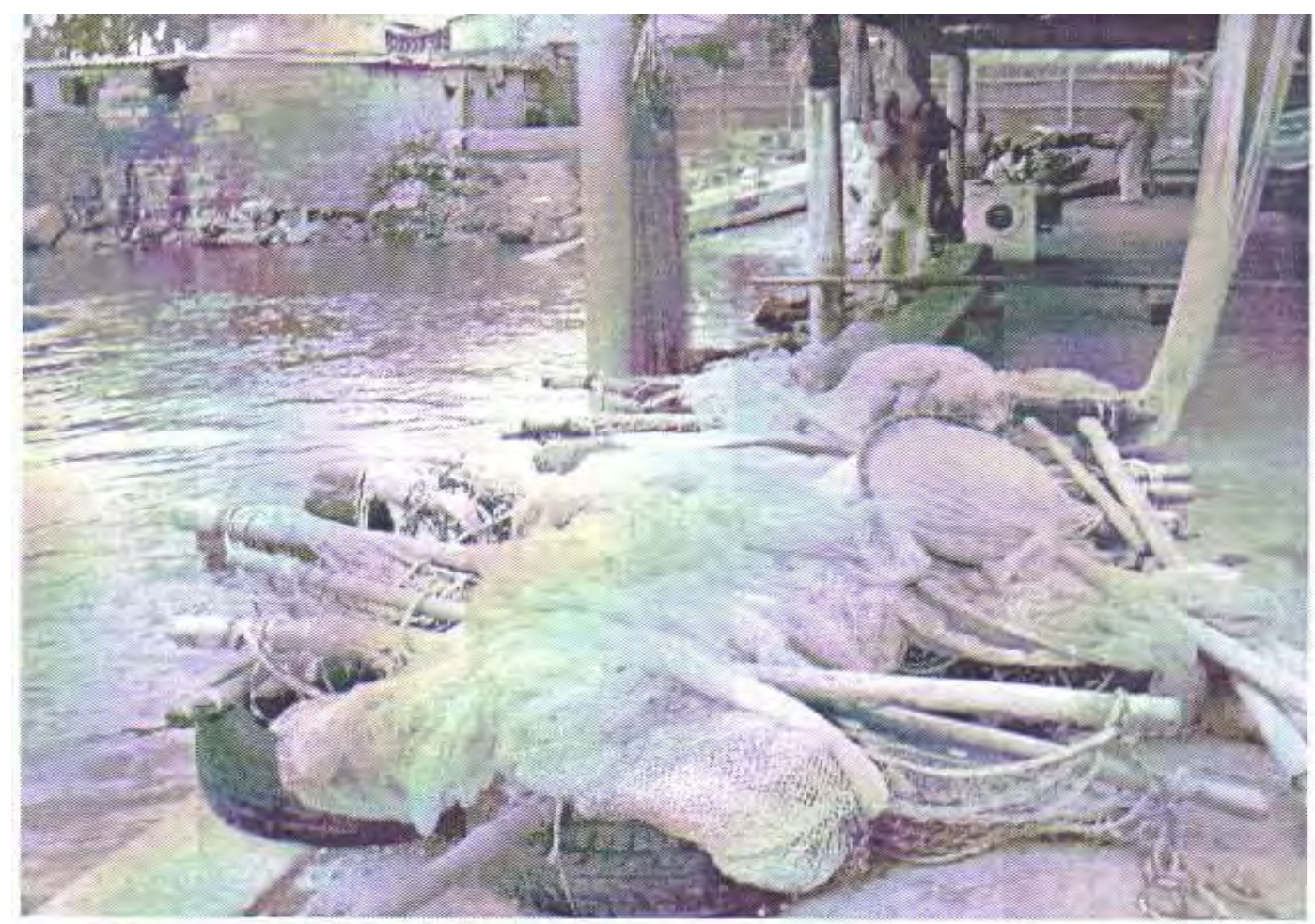

(Photo 6)

Photo 5 \& 6 : Filets éperviers séchés sous le $2^{\text {ème }}$ pont (Photo 5 ) et Filet fixe Avè stockés au campement de pêche de Placodji (jetéé) (Photo 6) (Badahoui, 21 février 2008). 


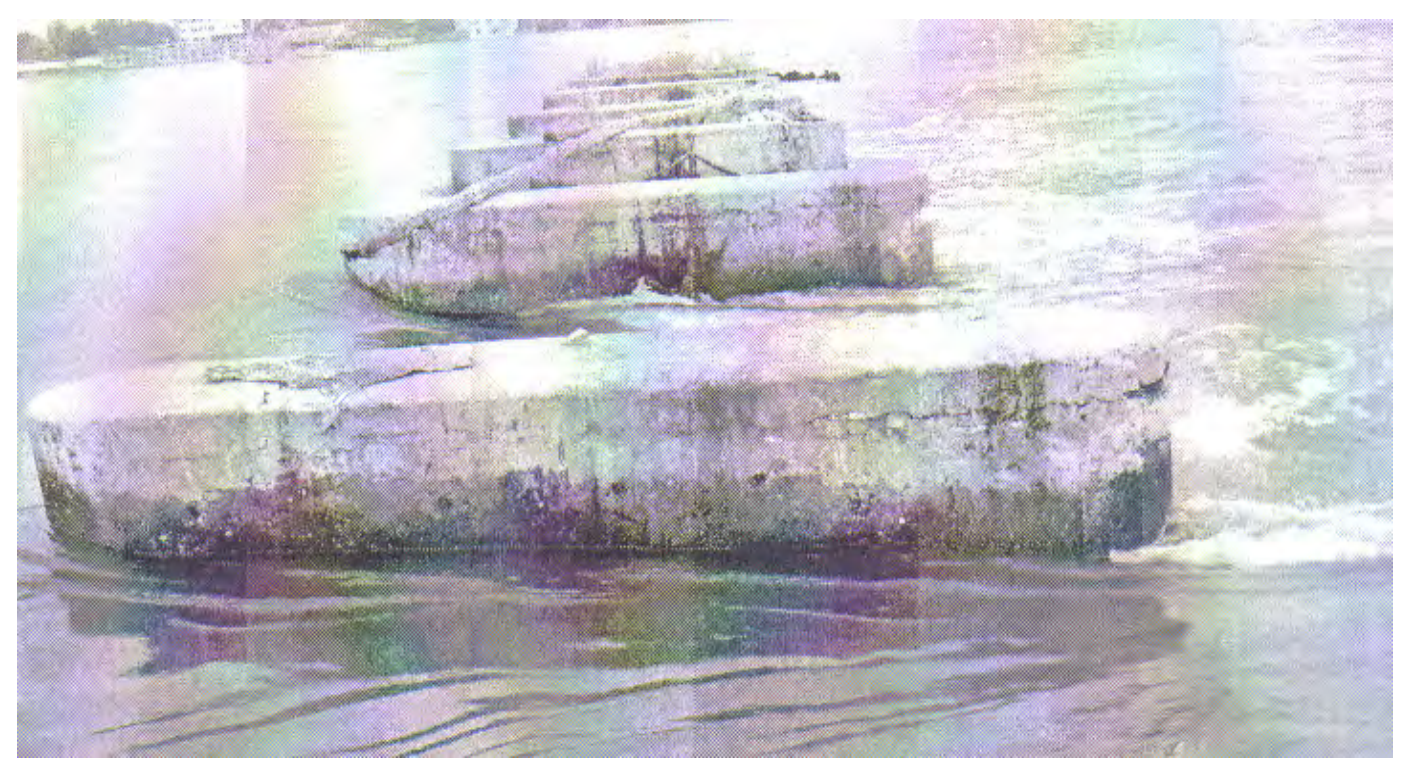

Photo 7 : Barrage de Cotonou (Badahoui, 21 février 2008).

compromis. Ces campements sont installés sur des immondices et constituent des zones insalubres dont l'occupation est formellement interdite par les pouvoirs publics. Nous avons dénombré onze (11) campements de pêches dans les localités riveraines du chenal. Il s'agit des campements de Dancodji (Akpakpa Dodomé); Placondji- jetée; Ancien pont (situé entre l'ancien et le 3è pont et la Direction des Pêches); Abokicodji lagune; Dédokpo (situé entre yatch club et le $2^{\text {ème }}$ pont); Kpankpan; Midombo ; Adogléta ; Agbato; Minontchou et, Ladji. Nous avons recensé au total dans le chenal, 270 pirogues de pêches (les unes accostées dans les campements de pêche, les autres opérant dans le plan d'eau du chenal), 22 parcs acadja, 352 pieux pour filet fixe (appelé Avè en langue locale), 28 filets éperviers (appelé Djètowlé en langue locale), et 14 nasses à crevettes (une pêcherie sédentaire conçue spécifiquement pour pêcher les crevettes; les pêcheurs y accrochent des lampions la nuit qui attirent les crevettes qui se font emprisonner dans les poches de l'engin).

La présence en permanence des engins et méthodes de pêche recensés signalent que la pêche s'exercent effectivement dans le chenal, alors que cette activité est interdite par les pouvoirs publics. Dans ce cas, le rôle d'échange biologique entre mer et lagune que doit jouer le chenal ne sera-t-il pas compromis? Ne va-t-on pas assister à la rareté ou la disparition de certaines espèces migratrices? La pêche dans le chenal n'estelle pas préjudiciable à la faune ichtyologique?

\section{Evolution de la faune ichtyologique}

Avant 1885, la diversité spécifique du lac Nokoué était estimée à 153 espèces de poissons réparties dans 40 familles (Roche International, 2000).

De l'aménagement du port de Cotonou à l'installation du barrage, la diversité spécifique du lac Nokoué et du chenal de Cotonou a connu une réduction drastique du nombre des espèces de poissons présents dans les plans d'eau de Cotonou. Il a été dénombré 73 espèces de poissons réparties dans 35 familles (Roche International, 2000). De l'installation du barrage à la fermeture complète par banc de sable, la diversité spécifique du lac Nokoué et de la lagune de Cotonou est passée à 20 espèces de poissons réparties dans 15 familles.

Bourgoignie (1972) a compté au total dans le milieu ichtyologique du terroir lacustre, une centaine d'espèces de poissons et de crustacés, mais une dizaine seulement a une réelle valeur économique et représente la quasi-totalité du tonnage pêché. D'une façon générale, le nombre d'espèces de poissons n'a 
cessé de baisser d'une période à une autre. Autrefois, avant que les effets de l'ouverture permanente du chenal n'apparaissent, deux espèces, à elles seules, Tilapia heudeloti et Ethmalosa fimbriata constituaient $70 \%$ des captures (Bourgoignie, 1972). Depuis lors, il y a une évolution défavorable de la composition qualificative $\mathrm{du}$ fait de la diminution $\mathrm{du}$ pourcentage des Tilapia, Ethmaloses, Mugilidae, qui représentait $77 \%$ des poissons capturés au filet épervier (Bourgoignie, 1972). Les poissons divers, la plupart d'origine marine et moins intéressants dans l'exploitation économique de la lagune, sont deux fois plus nombreux qu'avant. Notons que le peuplement du lac est le fait d'un mélange d'espèces d'eau douce pouvant supporter une variation considérable de la salinité et des espèces marines. Ce mélange est très peu diversifié et se compose essentiellement de Tilapia, d'Ethmaloses, de Mugil, de Chrysichthys, d'Elops et de quelques autres espèces épisodiques (Baglo, 1994).

Entre 1957 et 1959, les Tilapia, Ethmalose, Mugilidae constituaient pour le lac Nokoué $77,2 \%$ des captures au filet. Ces espèces sont surtout des phytoplanctonophages. En période de fermeture du chenal de Cotonou, on enregistre une forte proportion de Tilapia. Ceci confirme l'hypothèse des pêcheurs selon laquelle le Tilapia trouve son milieu favorable de reproduction et de croissance en lagune fermée (Baglo, 1980).

Les études de Van Thielen et al. (1993) ont montré que la faune ichtyologique qui constitue l'unique ressource des "Toffinu" du lac Nokoué est abondante et variée. Elle compte 38 familles et 75 espèces d'eau douce ou d'origine marine. Mais seulement une dizaine a une valeur économique. Trois espèces dominent essentiellement sur le plan quantitatif: les tilapias (appelé en Goun : Wè), les Ethmaloses (Tchèkè en Goun) et les crevettes (Dégon en Goun).

Spécialement dans le chenal de Cotonou, il faut remarquer la présence de quatre familles de poissons et de crustacés. Il y a: les Claroteidae (Chrysichthys auratus, Chrysichthys nigrodigitatus); les Cichlidae (Hemichromis fasciatus, Sarottherodon melanotheron, Tilapia guineensis); les
Gecarcinidae (Cardiosoma armatum) et les Palaemonidae (Macrobrachium sp).

En dehors de ces poissons et crustacés, on note l'abondance relative des crustacés constitués de crevettes surtout Panaeus duorarum qu'on retrouve surtout dans l'Ouest du lac Nokoué, la partie la plus sale et la plus vaseuse. Vers l'Est, les crevettes sont de plus en plus rares, au fur et à mesure que l'on arrive dans le canal de Totchè. Le lac renferme aussi des crabes (Callinetes latimanus).

\section{Impacts des activités de pêche sur les ressources halieutiques}

Les activités de pêche sur le chenal de Cotonou ont un impact négatif certain sur l'environnement aquatique. Il faut rappeler que le chenal de Cotonou joue un rôle d'échange biologique entre la mer et le lac Nokoué. Cette fonction est déjà compromise par la pratique de certains engins de pêche non sélectifs (filets à mailles fines) qui ne favorisent pas la migration des espèces selon leur cycle de reproduction de la mer vers le lac et vice-versa. Certains pêcheurs ont réussi à creuser des brèches sous la digue du barrage en enrochement pour poser leurs filets en vue de capturer les espèces qui migrent de la mer vers le lac Nokoué, en passant dans le chenal. D'autres pêcheurs ont péri dans cette pratique à grand risque sous l'effet des forts courants des marées. La ruée des pêcheurs professionnels sur le chenal a occasionné la surexploitation de la ressource halieutique ce qui a pour conséquence la dégradation à court, moyen et long termes de la base de la ressource. L'utilisation des engins de pêche non sélectifs favorise la détérioration de l'habitat de la faune aquatique à travers la capture systématique des juvéniles.

La pêche est donc interdite dans le chenal pour préserver de façon durable la faune ichtyologique. Il est aussi important de préserver les ressources halieutiques contre le contact avec les produits pétroliers déversés dans le chenal.

\section{Dégradation du chenal due au transport illicite des produits pétroliers}

Le chenal de Cotonou est navigable pour le transport des biens et des personnes d'une rive à l'autre et sur le cours du chenal.

Le transport fluvial des biens et des personnes n'a pas un impact direct sur 
l'environnement aquatique, mais le risque de dégradation de l'écosystème est grand quant à la pollution chimique des eaux par le déversement des produits pétroliers en provenance du Nigéria.

En effet, dans le secteur informel, les produits pétroliers sont illicitement transportés par voie fluviale et débarqués en vrac la nuit par centaine de bidons dans les embarcadères non aménagés. Ces produits pétroliers qui sont fréquemment déversés dans l'eau lors des manipulations constituent une source de pollution chimique du plan d'eau. Ainsi, la pollution peut toutefois contaminer le substrat et même annihiler les ressources halieutiques qui portent dans leur chair les métaux lourds dangereux. Les populations consommatrices des poissons pêchés dans les eaux polluées seront également contaminées par intoxication alimentaire.

Nous pensons enfin, à l'impact que pourra avoir l'extraction du sable lagunaire sur le fonctionnement hydrologique du chenal.

\section{Dégradation du chenal due à l'extraction de sable lagunaire}

Nous avons identifié au cours de notre enquête du 02 février 2008, une carrière de sable lagunaire devant le quartier Ladji à la sortie du chenal de Cotonou. Cette activité commerciale consiste à prélever artisanalement du sable lagunaire que les populations achètent pour les travaux de construction. Malheureusement, aucune étude n'est encore réalisée sur l'exploitation de cette carrière pour déterminer les dégradations qu'elle occasionne pour le lac et le chenal.

En nous inscrivant dans l'hypothèse du dragage de cette portion du lac, on peut supposer un instant que le prélèvement du sable permettra d'atténuer l'effet du comblement, tout en améliorant la bathymétrie et le régime hydraulique du plan d'eau. L'amélioration des conditions hydrologiques est un atout qui concoure à l'augmentation de la productivité halieutique.

Il s'avère donc nécessaire d'entreprendre des études dans ce sens pour connaître avec précision l'impact réel de l'extraction du sable sur le fonctionnement du chenal et sur les ressources aquatiques.

\section{Conclusion}

Au terme de cette étude, nous avons diagnostiqué les principaux facteurs de dégradation du chenal de Cotonou. $\mathrm{Au}$ nombre de ces facteurs, on peut citer entres autres :

- la pollution sous toutes ses formes qui affecte la qualité des eaux ;

- les conséquences de la construction du port de Cotonou en eau profonde avec l'ouverture permanente $\mathrm{du}$ chenal ayant entraîné d'importantes modifications hydrobiologiques et socioéconomiques ;

- les conséquences de la construction du barrage de Cotonou qui présente un défaut technique dans le mécanisme de réglage des échanges mer-lagune ;

- l'exercice des activités de la pêche malgré son interdiction dans le chenal par les pouvoirs publics.

Pour remédier à cette situation aggravante qui menace l'équilibre biologique de ce chenal, nous proposons les actions diligentes suivantes :

Engager la lutte contre la pollution des eaux Il s'agira pour l'Etat Béninois et la municipalité de Cotonou en associant les Organisations non Gouvernementales (ONG) impliquées de :

- faire une bonne gestion des déchets solides et liquides dans les quartiers riverains du chenal et en particulier au marché Dantokpa. La société de gestion des marchés (SOGEMA) a installé sur la berge du chenal en face du marché Dantokpa quelques poubelles mobiles (Photo 8). Cette action salutaire mérite d'être généralisée sur les deux rives du chenal et que les populations riveraines soient suffisamment sensibilisées à l'usage de ces poubelles ;

- détruire les latrines flottantes en construisant des latrines publiques améliorées sans communication avec le chenal. Une ONG de la place a construit sur la berge du marché Gbogbanou une latrine publique pour soulager les populations riveraines qui peinent beaucoup avant de satisfaire leurs besoins. Il revient aux gestionnaires des marchés riverains surtout les élus locaux, de sensibiliser les populations à faire un bon usage de cette infrastructure qu'il faut multiplier sur les deux rives du chenal ; 


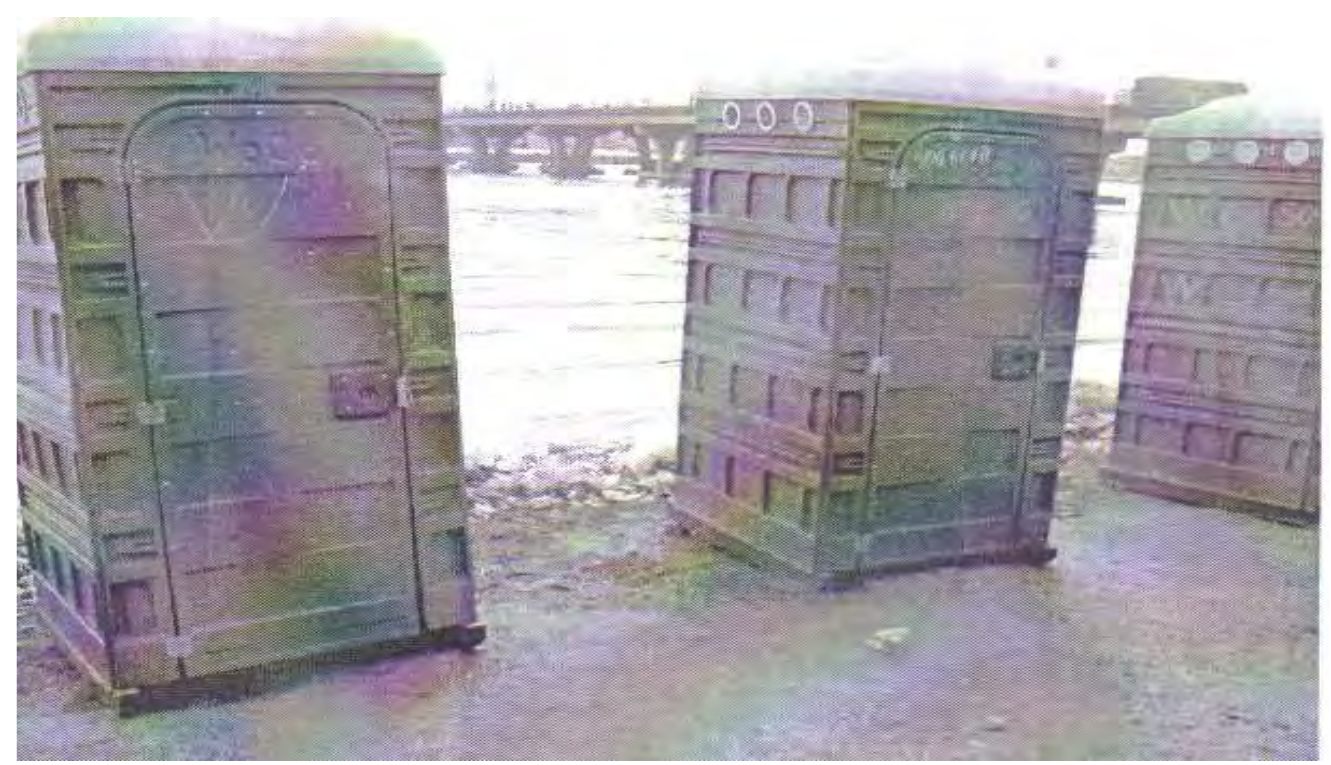

Photo 8 : Poubelles mobiles installées sur la berge du chenal devant le marché Dantokpa (Badahoui 21 octobre 08).

- traiter les eaux usées rejetées dans le chenal et créer des stations d'épuration de ces eaux usées ;

- lutter contre la pollution du chenal par les hydrocarbures en sensibilisant les populations notamment les gens qui transportent ces produits par voie fluviale. Les unités des Forces Navales se chargeront de renforcer les mesures de surveillance sur tout le plan d'eau afin de réduire les risques de pollution par le transport des produits dangereux. La présence des unités des Forces Navales au quartier Ladji est une solution idoine pour lutter contre la pollution du chenal par les hydrocarbures. Il faut donc doter ces unités des moyens pour accomplir efficacement leur mission.

\section{Assurer la régulation des échanges mer-} lagune.

Les pouvoirs publics, avec l'aide des Partenaires au Développement doivent entreprendre :

- la réhabilitation du barrage de Cotonou dont la crête devra être arasée à la côte de $+0,635$ mètres hydro ;

- la construction d'un épi Ouest pour assurer une section d'écoulement suffisante et stable de l'ordre de $500 \mathrm{~m}^{2}$.

\section{Sensibiliser les populations pour le respect de la loi cadre sur l'environnement}

A ce sujet, l'obligation sera faite à tout promoteur de réaliser d'abord les études d'impacts environnementales avant toute installation d'infrastructures à caractère social, écologique ou économique sur le lac, dans le chenal ou dans leur voisinage immédiat.

\section{Stabiliser les berges du chenal}

Les autorités administratives en charge de l'environnement et de l'urbanisme devront étudier et choisir le type d'ouvrage approprié qui tienne compte des conditions de reproduction de la faune aquatique. Les hangars avec terrasses bétonnées érigés en face du marché Dantokpa sont les types d'ouvrages construits pour stabiliser les berges dans cette partie du chenal. Pour freiner les effets de comblement du plan d'eau, il est souhaitable de stabiliser les deux rives du chenal en y installant des ouvrages de ce genre (Photo 9).

\section{Envisager la mise en place d'un ouvrage de contrôle à la sortie du chenal de Cotonou}

Le rôle de cet ouvrage dont la maîtrise d'œuvre sera étudiée avec les techniciens 


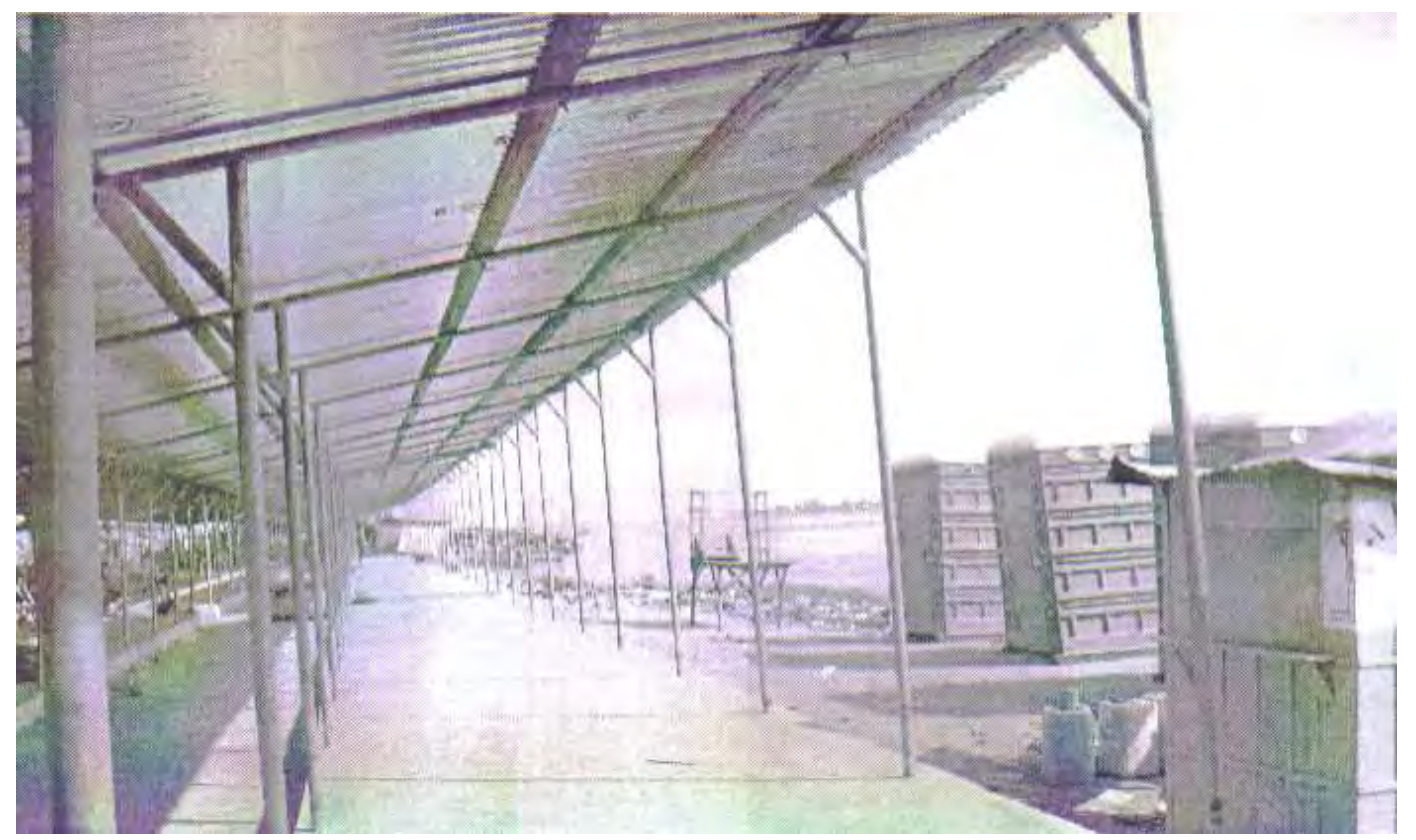

Photos 9: Construction de hangar avec terrasses bétonnées en face du marché Dantokpa (Badahoui 21 octobre 08$)$.

expérimentés, serait de contrôler le régime salin dans le lac Nokoué en vue :

- de dulcifier au maximum durant la saison sèche avec des secteurs orientaux de l'ordre de $2 \%$ o suivant le schéma proposé par Roche International (2000), pour favoriser la production halieutique d'eau douce ;

- d'inonder le maximum des plaines à Paspalum à la périphérie en crue ;

- d'éviter les inondations dans la ville, le maximum permis étant de l'ordre de 1,20 m et le niveau exceptionnel de $1,4 \mathrm{~m}$ selon la norme de SNC- LAVALIN proposée par Roche International (2000) ;

- de permettre la migration de certaines espèces et le passage d'embarcations aux étales de marée.

Faire recaser sur un autre site approprié les populations installées sur les berges du chenal

L'occupation anarchique des berges du chenal par certaines personnes au mépris des textes de réglementation en vigueur au Bénin, est un facteur qui favorise le comblement du plan d'eau. C'est pourquoi les autorités municipales en charge des affaires domaniales devront prendre toutes les dispositions pour installer les populations concernées ailleurs sur des espaces viabilisés.

Interdire la pêche dans le chenal de Cotonou

Depuis longtemps, les pêcheurs s'opposent farouchement à l'application de l'Arrêté N ${ }^{\circ} 068 / \mathrm{MDR} / \mathrm{DC} / \mathrm{CC} / \mathrm{CP} /$ du 12 mars 1997, portant réglementation de la pêche sur le complexe Delta de l'Ouémé - lagune de Porto-Novo - lac Nokoué). Or, cet arrêté a été pris pour favoriser la migration dans les deux sens des espèces halieutiques et surtout pour assurer la préservation des frayères en vue du repeuplement naturel du lac Nokoué qui communique avec la mer par le chenal de Cotonou. Mais force doit rester à la loi si on veut vraiment rétablir l'équilibre écologique du chenal. C'est pourquoi les pouvoirs publics doivent trouver à ces pêcheurs des mesures d'accompagnement avant de prétendre 
appliquer avec succès cette réglementation. C'est l'occasion pour l'administration des pêches d'instituer une police de pêche et la doter des moyens adéquats pour mieux accomplir cette mission régalienne qui est le suivi et le contrôle des plans d'eau en matière de pêche. Il est indiqué d'installer une seconde base des unités des Forces Navales à l'embouchure au quartier Placondji qui viendrait en renfort à la police de pêche pour dissuader les pêcheurs récalcitrants et faire respecter la réglementation des pêches dans le chenal.

\section{REMERCIEMENTS}

Nous remercions toutes les personnes ressources rencontrées dans le cadre de cette étude qui nous ont fourni des informations sur le terrain et de la documentation. Nos remerciements vont également à tout le personnel de la Direction des pêches du Bénin, particulièrement la Directrice de cette institution qui a mis à notre disposition une barque motorisée pour effectuer les sorties sur le chenal de Cotonou.

\section{REFERENCES}

Abou Y, Fiogbé ED, Micha J-C. 2007. Effect of stocking density on growth, yield and profitability of farming Nile tilapia, Oreochromis niloticus L., fed Azolla diet, in earthen ponds. Aquaculture Research 38: 595-604.

Belaud A.1987. Les eaux continentales : quantité et qualité. Cours DEA et formation continue. ENSA Touloue/ Ichtyologie appliquée, pp 76.

Boko M. 2004. La pollution urbaine à Cotonou (République du Bénin, Afrique occidentale). Publication de l'AIC, 4: 5561.

Boko M. 2004. Pollution de l'Environnement et Santé Publique (Edition revue et corrigée). UAC/CIFRED; $160 \mathrm{p}$.

Bonou C, Adisso P. 2002. Evaluation de la pollution organique et bactériologique due aux excrétas, aux eaux usées et aux déchets solides dans la lagune de Cotonou. CPU/UAC 2002, 66 p.

Bourgoignie G. 1972. Les Hommes de l'Eau, Ethno-écologie du Dahomey Lacustre. Editions Universitaires ; 391 p.

Colas R. 1976. La pollution des eaux. PUF (Que sais-je, 903).

Crosnier A, Berrit GR. 1963. Fonds de pêche le long des côtes des Républiques du Dahomey et du Togo. ORSTOM, Pointe Noire.

Dejoux C. 1988, La pollution des eaux continentales africaines, expériences acquises, situation actuelle et perspectives. ORSTOM, Paris, 513 p.

Furon R. 1963. Le problème de l'eau, l'eau dans la biosphère (océans et eau douce), l'eau et l'agriculture, les méfaits de l'eau, l'eau et l'industrie, l'eau dans les grandes villes. Payot, Paris, 251 p.

Fiogbé ED, Akitikpa B, Accodji J-M M. 2009. Essai de mise au point de formules alimentaires à base d'azolla (Azolla microphylla Kaulf) et de sous-produits locaux pour la pisciculture rurale $\mathrm{du}$ tilapia, Oreochromis niloticus L. Int. J. Biol. Chem. Sci., 3(2): 398-405.

Gaujous D. 1995. La Pollution des Milieux Aquatiques: Aide Mémoire (2è édn technique et documentation). Lavoisier ; $220 \mathrm{p}$.

Guilcher A. 1959. Précis d'Hydrologie Marine et Continentale ( $2^{\text {ème }}$ édn). Masson et Cie : Paris VI ; 344p.

Lalèyè P. 1995. Ecologie comparée de deux espèces de Chrysischtys, poissons siluriformes (Claroteidae) du complexe lagunaire lac Nokoué-lagune de PortoNovo au Bénin. Thèse de doctorat, Université de Liège, Belgique, 152 p. + annexes.

Larousse 2007. Dictionnaire illustré de 2007.

Le Barbe L. 1993. Les ressources en eaux superficielles de la République du Bénin. ORSTOM. Institut Français de Recherche Scientifique pour le Développement en Coopération, pp.25-73. 
Leite EC. 2002. Incidences de la dynamique des interventions humaines sur les écosystèmes naturels : le cas du complexe lac Nokoué - lagune de Porto-Novo au Bénin. Dire (La Revue des Cycles Supérieurs de l'Université de Montréal), 11(2).

Martin G. 1985. Point sur l'Epuration et le Traitement des Affluents: Eau, Air. Première Partie: Bactériologie des Milieux Aquatiques, Aspects Ecologiques et Sanitaires (vol. 2). Lavoisier: Paris ; $322 \mathrm{p}$.

Pliya J. 1980. La pêche dans le Sud - Ouest du Bénin. Etude de géographie appliquée sur la pêche maritime et continentale, AGECOP, Paris ACCT, 296 p.

Roche International. 2000. Etude du Projet d'aménagement des plans d'eau du Sud-
Bénin : l'Environnement, les eaux et les forêts, Volume 2- Tome 4 janvier.

Roche International. 2000. Etude du Projet d'aménagement des plans d'eau du SudBénin : Le secteur des pêches, Volume 2Tome 2, janvier.

Troadec JP. 1990. L'Homme et les Ressources Halieutiques: Essai sur l'Usage d'une Ressource Commune Renouvelable. IFREMER : Paris, 817 p.

Van Thielen R. 1996. Le rôle du système acadjas dans la gestion des eaux intérieures du sud-Bénin. PPL/GTZ/DP ; Bénin. 